\title{
Mathematical Model of Plates and Strips Rolling for Calculation of Energy Power Parameters and Dynamic Loads
}

\author{
Elena Sorochan ${ }^{1}$, Viktor Artiukh ${ }^{2, *}$, Boris Melnikov ${ }^{2}$, and Talzhan Raimberdiyev ${ }^{3}$ \\ ${ }^{1}$ Pryazovskyi State Technical University, Universytets'ka, 7, Mariupol, 87500, Ukraine \\ ${ }^{2}$ Peter the Great St. Petersburg Polytechnic University, Polytechnicheskaya, 29, Saint-Petersburg, \\ 195251, Russia \\ ${ }^{3}$ Hoja Akhmet Yasawi International Kazakh-Turkish University, Sattarkhanova, 29, Turkestan, \\ 161200, Kazakhstan
}

\begin{abstract}
Presents the results of studies of innovative materials in the field of metallurgy. The article describes mathematical models for calculating energy-parameters of hot rolling plates and strips using a more correct evaluation of external friction, which is necessary for precisely determining the values of the dynamic loads in the drive's line of rolling mills stands. The results can be used for designing rolling mills drive lines' mechanical facilities.
\end{abstract}

\section{Introduction}

Iron and steel machines have to work under heavy dynamic modes, so requirements for design strength and reliability are high. The quality of these machines depends largely on how precisely their main parameters were calculated. At the same time, a machine must be considered as it actually is, i.e. with due regard to the material properties (elasticity), the actual character of application of external loads, taking into consideration the motor's characteristics and gaps in the drive lines, as well as the system's dissipative properties. Rolling machines typically have the heaviest dynamic modes; the character of their loads application is actually close to that of impact character. Thus, if dynamic excitation is neglected, it could lead to great errors in calculations for iron and steel machines. Dynamic models of various complexity levels are used for investigating the phenomena occurring in the main line of a rolling mill, when rolled metal is gripped by the rollers. The existing dynamic models have a drawback: a simplified mathematical model of the rolling process [1]. In some cases, the same simplified models of linear dependence of rolling momentum and force in time are used [2]. These simplifications lead to errors in calculations. Therefore, it is vital that we adapt the existing models of the process to dynamic calculations of the main line of a rolling mill.

\footnotetext{
Corresponding author: artiukh@mail.ru
} 


\section{Materials and Methods}

As a rule, time is a variable in dynamic models, so the problem is restricted to determining geometrical, kinematic and energy-power parameters of the rolling process at any time instant of metal filling the deformation area [3]. At strict target setting, the solution is reduced to the solution of a third degree equation. However, for determining kinematic and geometrical characteristics of the area of deformation, we can use the following dependence [4]:

$$
V_{x}=V_{0}+\left(\frac{\tau_{x}}{\tau_{0}}\right)^{2}\left(V_{1}-V_{0}\right)
$$

where $\mathrm{V}_{0}, \mathrm{~V}_{\mathrm{x}}$ - is the velocity of rolling at the entrance to the area at a current instant $\tau_{\mathrm{x}}$, respectively; $\tau_{0}$ - time of metal gripping by the rolls, equal to

$$
\tau_{0}=2 \sqrt{R\left(h_{0}-h_{1}\right)} /\left(V_{0}+V_{1}\right)
$$

$\mathrm{R}$ - is the rolls radius; $\mathrm{V}_{1}$ - the velocity of rolling at the exit from deformation area; $\mathrm{h}_{0}, \mathrm{~h}_{1}-$ are the initial and final thickness of the rolled stock.

The values of the rolled stock thicknesses at the current instant can be found from the law of constancy of the seconds volumes [5]:

$$
h_{x}=h_{0} V_{0} / V_{x} .
$$

Further calculations are made in accordance with one of the following methods of calculation, depending upon the value of the relation $L / h_{c p}$.

Now, let us consider the engineering methods of determining energy parameters for hot rolling relatively thin sheets and strips, i.e. at $L / h_{c p}>2,5[6]$.

Having made in accordance with the methods applied in $[7,8]$ division of the integral area of deformation (Fig. 1, a) into the area of tardiness and the area of advance, with the lengths $\mathrm{L}_{\text {от }}$ and $\mathrm{L}_{\text {оп, }}$ respectively, we will consider the condition of static equilibrium, detached within the tardiness area of elementary metal volume (Fig. 1, b) at projecting of all exerted forces at $\mathrm{x}$-horizontal axis $\mathrm{x}$ :

$$
\sigma_{x} h_{x}-\left(\sigma_{x}+d \sigma_{x}\right)\left(h_{x}+d h_{x}\right)+2 \mathrm{p}_{\text {хот }} d x \sin \alpha_{x} / \cos \alpha_{x}-2 \tau_{x} d x \cos \alpha_{x} / \cos \alpha_{x}=0,
$$

where $\mathrm{p}_{\mathrm{x}}, \sigma_{\mathrm{x}}, \tau_{\mathrm{x}}$ - the current values of normal contact axial and tangent contact stresses, compression stresses are taken for positive values $\sigma_{\mathrm{x}}$ and $\tau_{\mathrm{x}}-$ stresses with vector orientation with transfer of the rolled metal; $h_{x}, \alpha_{x}$ are the current values of the thickness of the rolled strip and contact angles, the numerical values of which at approximation of contact $\operatorname{arcs}$ with chords are equal $h_{x}=h_{1}+\Delta h x / L$ and $\alpha_{x}=\alpha=\operatorname{arctg}(0,5 \Delta h / L)$. 


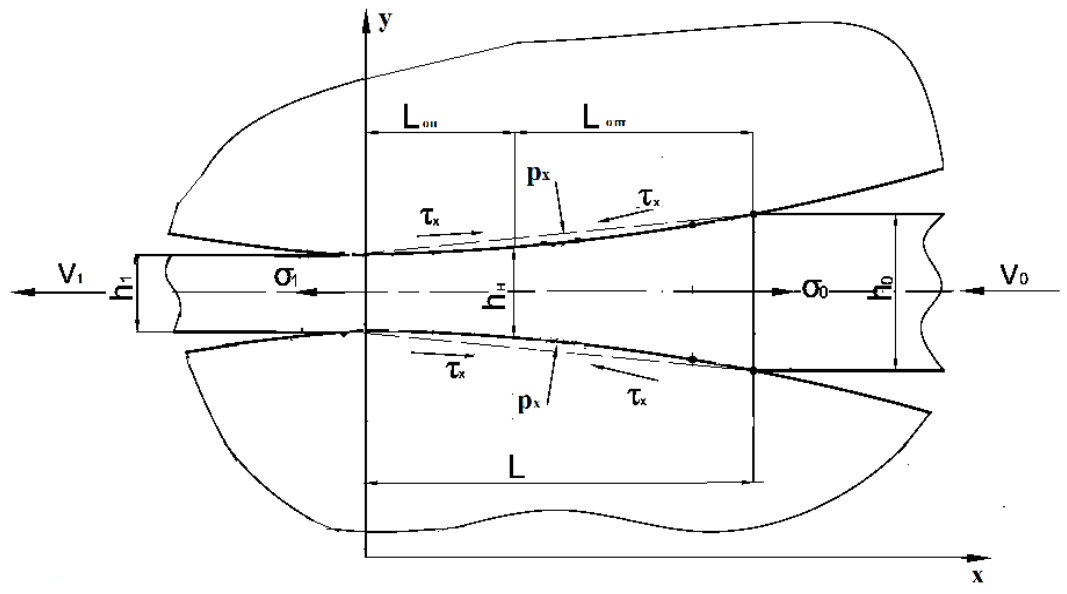

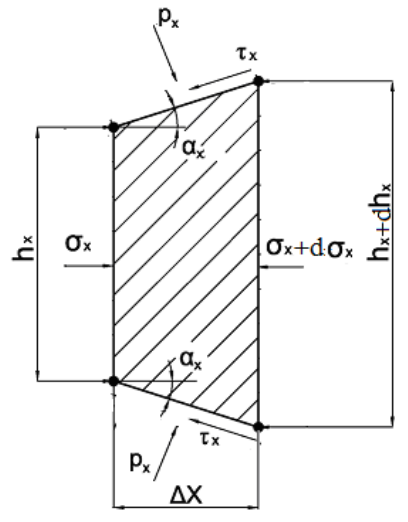

b)

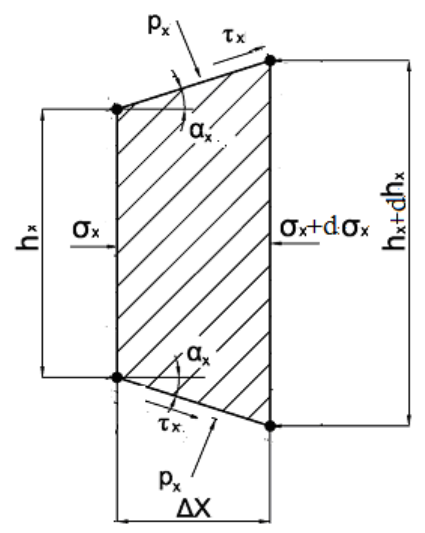

c)

Fig. 1. Computational models of the integral focal point of the deformation (a) as well as elementary volumes of metal distinguished in the slower zone (b) and the faster zone (c), as applied to the engineering mathematical simulation of energy parameters of the process of hot symmetrical rolling of relatively thin strips.

Taking into consideration the law of external contact friction $\tau_{x}=2 K_{c} \mu$ (Siebel's law), recommended by the authors [9] $d \sigma_{x} d h_{x} \approx 0$ and disregarding infinitely small values of the second order along with taking into account the obvious purely geometrical relations $2 d x \sin \alpha_{x} / \cos \alpha_{x}=2 d x t q \alpha=d h_{x} \quad$ и $d x=d h_{x} /(2 t q \alpha)$ the differential equation (4) can be represented as:

$$
-\sigma_{x} d h_{x}-d \sigma_{x} h_{x}+\mathrm{p}_{\text {хот }} d h_{x}-2 K_{c} 2 \mu d h_{x} /(2 t q \alpha)=0
$$

where $\mu$ are coefficients of plastic friction, the values of which are supposed to be constant along the entire length of deformation area.

From the complete formula of description of the conditions of plasticity [9]:

$$
\left(p_{x}-\sigma_{x}\right)^{2}+4 \tau_{x y}^{2}=4 K_{c}^{2}
$$


and taking into account the results of two-dimensional analysis of metal stressed state [10, 11], according to which tangential components of stresses deviator $\tau_{x y}$ in the height of the deformation area sections are changed close to the linear, in compliance with the law, their average integral evaluation corresponds to the following equation:

$$
\tau_{x y c}=\tau_{x} / 2=2 K_{c} \mu / 2
$$

In the long run we will get the following for the values of normal contact stresses $\mathrm{p}_{\text {хот, }}$, occurring along the length of the area tardiness:

$$
\mathrm{p}_{\text {хот }}=2 K_{c}\left(\delta_{\mu}-a_{k}\right) \ln \left(h_{0} / h_{x}\right)+2 K_{c} a_{k}-\sigma_{0}
$$

where $a_{k}=\sqrt{1-\mu^{2}}$ is an auxiliary variable used for a simplified form of description; $\delta_{\mu}=2 \mu L / \Delta h$ is an auxiliary variable characterizing the influence of boundary conditions within the deformation area at the process of hot rolling.

Likewise, for the area of advance of deformation area (see Fig. 1, a, c) we get:

$$
\sigma_{x} h_{x}-\left(\sigma_{x}+d \sigma_{x}\right)\left(h_{x}+d h_{x}\right)+2 \mathrm{p}_{\text {хоп }} d x t q \alpha_{x}+2 \tau_{x} d x=0 .
$$

As a final view, with regard to the area of advance we will get:

$$
\mathrm{p}_{\text {хоп }}=2 K_{c}\left(\delta_{\mu}+a_{k}\right) \ln \left(h_{x} / h_{1}\right)+2 K_{c} a_{k}-\sigma_{1}
$$

Based on the condition of equality of normal contact stresses for the area of tardiness and the area of advance in the neutral section of deformation area $\left.\mathrm{p}_{\mathrm{xoT}}\right|_{\mathrm{h}_{\mathrm{x}}=h_{H}}=\left.\mathrm{p}_{\mathrm{xо \Pi}}\right|_{\mathrm{h}_{\mathrm{x}}=h_{H}}$ we will write the following:

$$
2 K_{c}\left(\delta_{\mu}-a_{k}\right) \ln \left(h_{0} / h_{H}\right)+2 K_{c} a_{k}-\sigma_{0}=2 K_{c}\left(\delta_{\mu}+a_{k}\right) \ln \left(h_{\mu} / h_{1}\right)+2 K_{c} a_{k}-\sigma_{1}
$$

from which the thickness of a strip in the $h_{H}$ section and the length of the area of advance $L_{a}$ (see Fig. 1, a) could be defined as:

$$
\begin{gathered}
h_{H}=\exp \left\{\left[\delta_{\mu} \ln \left(h_{0} h_{H}\right)-a_{k} \ln \left(h_{0} / h_{1}\right)+\sigma_{1} / 2 K_{c}-\sigma_{0} / 2 K_{c}\right] /\left(2 \delta_{\mu}\right)\right\}= \\
\left.=\sqrt{h_{0} h_{1}} \exp \left\{a_{k} \ln \left(h_{1} / h_{0}\right)+\sigma_{1} / 2 K_{c}-\sigma_{0} / 2 K_{c}\right) /\left(2 \delta_{\mu}\right)\right\} \\
\mathrm{L}_{\text {on }}=\left(h_{H}-h_{1}\right) L / \Delta h .
\end{gathered}
$$

As the result of integrating of the calculated in accordance with (8) and (10) distributions of normal contact stresses $\mathrm{p}_{\text {хот, }} \mathrm{p}_{\text {хоп }}$ along the entire area of deformation:

$$
\mathrm{n}_{\sigma}=(1 / L)\left[\int_{0}^{L_{o n}} \mathrm{p}_{\text {хоп }} d x+\int_{L_{\text {on }}}^{L} \mathrm{p}_{\text {хот }} d x\right]
$$


An average integral value of the coefficient of stressed metal state could be determined: $\mathrm{n}_{\sigma}=p_{c} / 2 K_{c}$, which may be necessary for evaluation of an average integral value of normal contact stresses $\mathrm{p}_{\mathrm{c}}$ and the value of the rolling force $\mathrm{P}=2 K_{c} \mathrm{n}_{\sigma} B L=p_{c} B L$

$$
\begin{aligned}
& n_{\sigma}=\frac{1}{L}\left\{\int_{0}^{L_{o n}}\left[\left(\delta_{\mu}+a_{k}\right) \ln \left(\frac{h_{1}+\Delta h x / L}{h_{1}}\right)\right] d x+\int_{0}^{L_{o n}}\left(a_{k}-\frac{\sigma_{1}}{2 K_{c}}\right) d x+\right. \\
& \left.+\int_{L_{o n}}^{L}\left[\left(\delta_{\mu}-a_{k}\right) \ln \left(\frac{h_{1}+\Delta h x / L}{h_{0}}\right)\right] d x+\int_{L_{o n}}^{L}\left(a_{k}-\frac{\sigma_{0}}{2 K_{c}}\right) d x\right\} .
\end{aligned}
$$

From this equation, with due regard to substitution of variables of integrating limits $\left(h_{1}+\Delta h x / L\right) / h_{1}=U_{1},\left(h_{1}+\Delta h x / L\right) / h_{0}=U_{2}$, and integrating itself, with subsequent mathematical transformation we will finally have:

$$
\begin{aligned}
& n_{\sigma}=\frac{1}{L}\left\{\int_{1}^{h_{\mu} / h_{1}} \frac{h_{1} L}{\Delta h}\left[\left(a_{k}+\delta_{\mu}\right) \ln U_{1}\right] d U_{1}+\left(a_{k}-\frac{\sigma_{1}}{2 K_{c}}\right) L_{o n}+\right. \\
& +\int_{h_{\mu} / h_{0}}^{1} \frac{h_{0} L}{\Delta h}\left[\left(a_{k}-\delta_{\mu}\right) \ln U_{2}\right] d U_{2}+\left(a_{k}-\frac{\sigma_{0}}{2 K_{c}}\right)\left(L-L_{o n}\right)= \\
& =\left(\delta_{\mu}+a_{k}\right)\left[\frac{h_{H}}{\Delta h} \ln \frac{h_{H}}{h_{1}}-\left(\frac{h_{H}-h_{1}}{\Delta h}\right)\right]+\left(a_{k}-\frac{\sigma_{1}}{2 K_{c}}\right)\left(\frac{h_{H}-h_{1}}{\Delta h}\right)+ \\
& +\left(\delta_{\mu}-a_{k}\right)\left[\frac{h_{0}-h_{H}}{\Delta h}-\frac{h_{H}}{\Delta h} \ln \frac{h_{0}}{h_{H}}\right]+\left(a_{k}-\frac{\sigma_{0}}{2 K_{c}}\right)\left(\frac{h_{0}-h_{H}}{\Delta h}\right) .
\end{aligned}
$$

The value of the aggregate rolling momentum, taking into consideration the opposite direction of the tangential contact stresses $\tau_{\mathrm{x}}$ in the areas of tardiness and advance (see Fig. 1), in this case corresponds to:

$$
M_{\Sigma}=2 \times 2 K_{c} \mu R B\left(L-2 L_{o n}\right)=2 \times 2 K_{c} \mu R B L\left[1-2\left(h_{H}-h_{1}\right) / \Delta h\right] \text {, }
$$

where $\mathrm{R}$ and $\mathrm{B}$ are the radii of the operating rolls and the width of the rolled strip.

On the whole, the presented dependences, combined with calculation of the average integral value of the doubled resistance to shift $2 \mathrm{Kgf}$, with regard to elastic compression of the operating rolls and the presence of the elastic recovery [10] area, made up a complete algorithm for mathematical engineering modelling of energy parameters of the process of hot rolling of relatively thin strips, with due regard to more precise character of distribution of the boundary conditions of deformation area.

Correction of the original prerequisites (concerning the conditions of plasticity and analytical description of the coefficient of external contact friction) allowed us to develop engineering methods of calculation of energy- parameters of the process of hot rolling of relatively thin strips, with the degree of their non-correspondence, as compared to more strict two-dimensional analysis methods in relative measuring, not having exceeded $5 \%$.

Let us now discuss the methods for determining the energy- parameters, in case of hot 
rolling of relatively medium and heavy plates and strips. i.e. at $L / h_{c p}<2,5$.

The current level of computer technologies make it possible to apply sophisticated computer programs, based on the Finite Elements Method (FEM). ABAQUS/CAE [12] shell being one of them, allows us to take into consideration external friction coefficient, apart from more precise calculations, unlike the existing engineering methods.

This mathematical model was realized with Abaqus 6.5 FEM modelling using ABAQUS $\backslash$ Standard [12] programme package. For calculations, the operating rolls were simulated by means of not-deformed analytical surfaces, in accordance with the established assumptions whereas metal was simulated by 4-nodal, two-dimensional elements, equipped with CPE4R destruction control. We ensured quick formation of the original data for calculations by creating a parameterized model. The values of the original strip's thickness, the strip's thickness after rolling, radii of the upper and the lower rolls were varied without rearrangement of the model, as well as external friction coefficients, angular velocities of the operating rolls rotation and also the number of fragmentations in the strip's length and thickness for automatic generation of the finite elements network. Mechanical properties of the band's material were introduced by the corresponding values of resistance to deformation, depending upon the degree and velocity of deformation and metal temperature, determined by Andreyuk's method [13].

Mathematical simulation of the process itself was carried out in two steps. First, the yield of the strip by the operating rolls being simulated. Second; rotation of the operating rolls with specified angular speed was evaluated and hence, the band's transfer along the operating line, i.e. the direct realization of the process of rolling.

With the help of ABAQUS $\backslash$ CAE package, the coefficient of the stressed state was calculated, being expressed from the formula of rolling strength:

$$
P=2 K_{c} L B n_{\sigma}
$$

where $2 \mathrm{~K}_{\mathrm{c}}$ is a doubled average integral value of resistance to frequent shift, determined in accordance with the methods, employed in L.V.Andreyuk's works [13], or M.Y.Browman's works [14]; B is the width of the rolled strip; $L=\sqrt{R \Delta h-\left(\Delta h^{2} / 4\right)}-$ general length of the area of plastic forming; $h_{0}, h_{1}, \Delta h=h_{0}-h_{1}$ being the thickness prior and after rolling and the value of absolute reduction; $\mathrm{R}$ is the radius of the operating rolls of the rolling mill.

At the specified value of the rolling force and varied values, the specified distributions of the stressed state coefficient $L / h_{c p}$ were determined, presented in Fig. 2 . Here, the obtained dependences were compared to the conventional methods $[15,16]$.

The reliability of the results obtained by FEM was confirmed by comparing it to the methods used in the works of V.F.Potapkin [17] and M.Y.Browman [14], with regard to determining arm's coefficient, exerting influence upon the value of the rolling momentum:

$$
M=2 \cdot 2 K_{c} n_{\sigma} L^{2} B \psi
$$

The values of arm's coefficient, expressed by the formula (19), for a known value of the rolling momentum $\mathrm{M}$, calculated by means of FEM [18, 19] and also based upon the aforesaid methods, depending on $L / h_{c p}$ is pictured in Fig. 3.

Proceeding from the specified distributions, given in Fig. 2 and Fig. 3, we may make conclusions regarding the reliability of the results obtained by FEM. This provides one of 
the most precise methods for the design of equipment and technologies of hot rolling mills. The generalized results for stressed state coefficient and arm's coefficient, determining the rolling momentum are represented in Fig. 4.

From the analysis of the represented specified distributions it becomes obvious that unlike the methods used in $[13,14,17]$, the influence of the coefficient of external friction $\mu$ is essential enough even in $L / h_{c p}>1$ range. Analytically, the results of the present investigation could be presented by the dependence with regard to coefficient of metal stressed $n_{\sigma}$ (see Fig. 4, a) state as follows:

$$
n_{\sigma}=1+0,65 \mu\left(L / h_{c p}-1\right)
$$

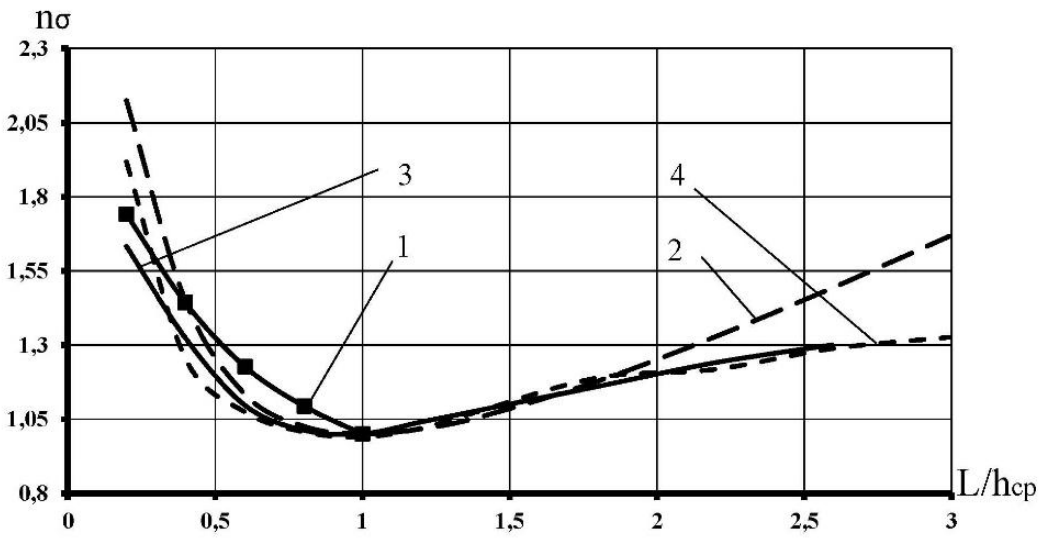

1 - Tselikov-Smirnov's methods [15]; 2 - M.Y.Browman's methods [14];

3 - V.Potapkin's methods [17]; 4 - numerical values, obtained by means of FEM.

Fig. 2. Specified distribution of coefficients of stressed state $\mathrm{n}_{\sigma}$ depending on the value $L / h_{c p}$, obtained on the basis of different methods.

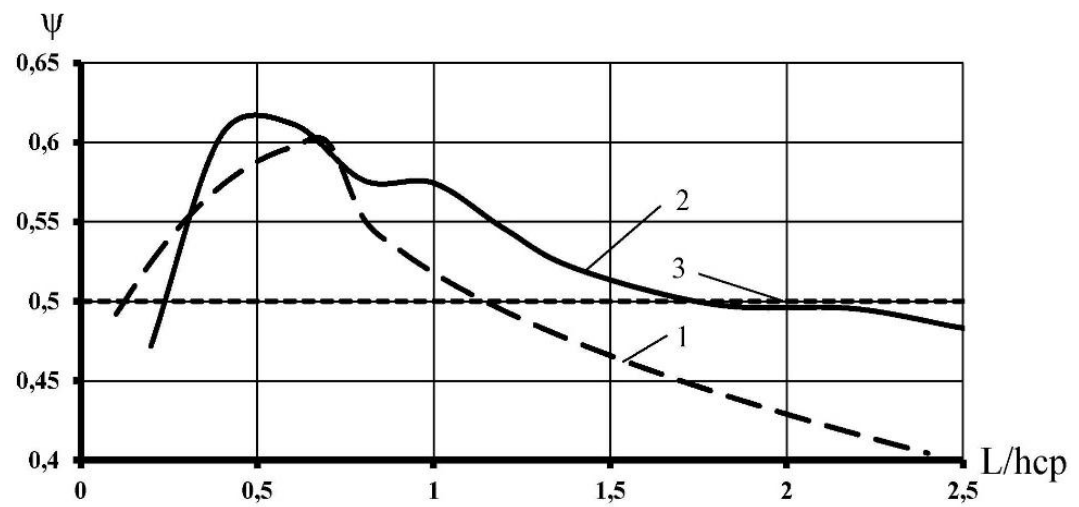

1 - V.F.Potapkin's methods [17]; 2 - numerical values, obtained by means of the method of finite elements; 3 - M.Y.Browman's methods [14].

Fig. 3. Estimated distribution of arm's coefficient $\psi$ depending on the value $L / h_{c p}$, obtained with application of different methods. 


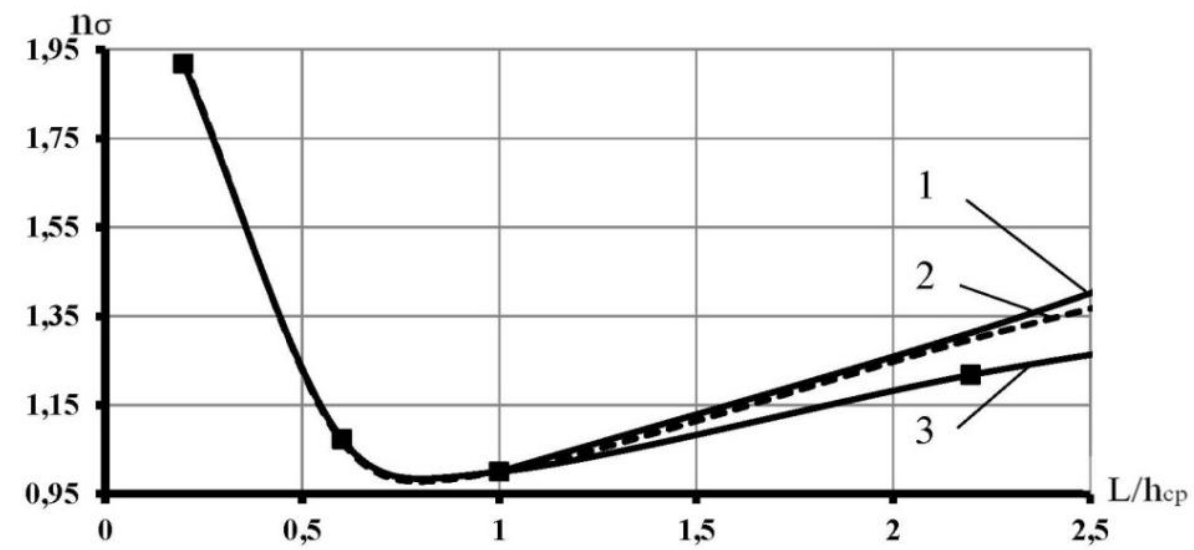

a)

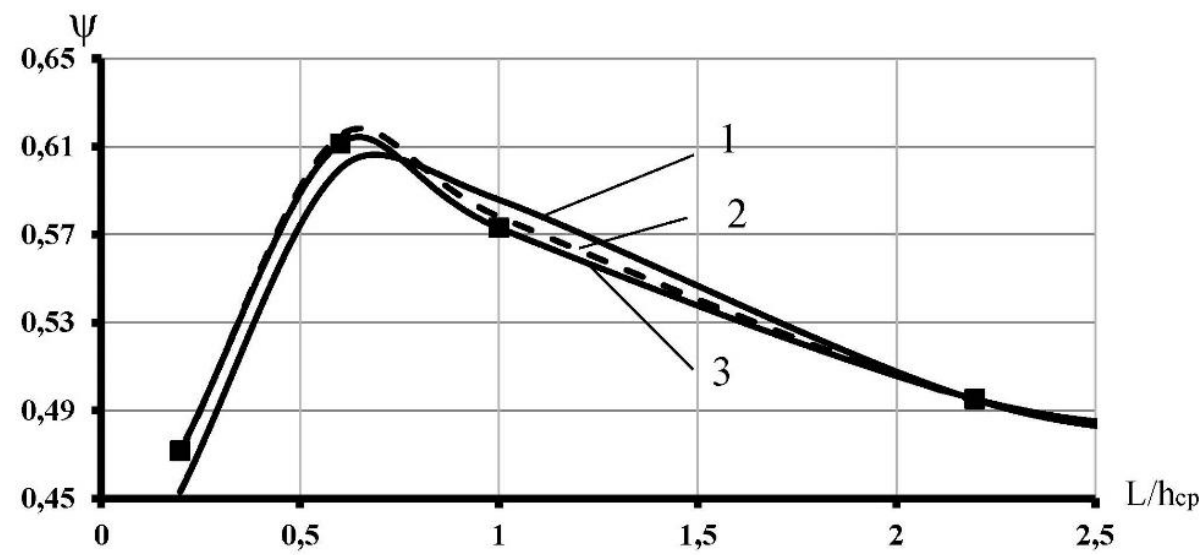

b)

$$
\mu=0,45-(1), \mu=0,35-(2), \mu=0,25-(3)
$$

Fig. 4. Estimated distributions of the coefficient of stressed state $n_{\sigma}(a)$ and arm's coefficient $\psi(b)$ depending upon the relation between the contact arc and the average thickness of the ingot within the area of deformation $L / h_{c p}$, obtained at different values of friction coefficient.

The influence of the forces of external friction $\psi$, upon arm's coefficient determining the rolling momentum is not so essential (see Fig. 4, b) and can be described by a single analytical dependence like:

$$
\psi=0,62-0,109\left(L / h_{c p}-0,6\right)+0,023\left(L / h_{c p}-0,6\right)^{2}
$$

Comparison of FEM to the existing methods of determination of the coefficient of stressed state helped to validate this method. At $L / h_{c p}<1$, the reliable interval lies within $0,996<X_{i}<1,004$ limits; the reliable interval at $1 \leq L / h_{c p} \leq 2,5$ lies within $0,95<X_{i}<1,03$ which fully confirms the reliability of the results obtained by FEM, namely by ABAQUS $\backslash$ CAE package.

The developed engineering mathematical model was used for calculations of external 
loads at rolling in the corresponding dynamic model.

The dynamic model was based on compilation of the system of differential equations of the motion of inertia masses of a mechanical system of an operating stand of a rolling mill. The stage, when metal is gripped by the rolls and the stage of metal rolling after gripping, was analyzed separately. In accordance with recommendations, in [16] a dependence for determination of elastic momentum in $i$ - phase of gripping was used:

$$
\begin{aligned}
& M_{12}=n_{2} M_{a}(1-\cos p t)+M_{n p}\left(1+\frac{\sin p t_{0}}{p t_{0}}\right)+ \\
& +n_{2} M_{n p}^{\prime}\left(\frac{\lambda^{2}}{1+\lambda^{2}} e^{-t / T_{M}}+\frac{\lambda \sin p t-\lambda^{2} \cos p t}{1+\lambda^{2}}\right)^{\prime},
\end{aligned}
$$

where $M_{a}=I_{0} \omega_{H} / \mathrm{t}_{\mathrm{p}}-M_{n p} \mathrm{~T}_{\mathrm{M}} / t_{0} ; M_{n p}^{\prime}=M_{n p} \mathrm{~T}_{\mathrm{M}} / t_{0} ; n_{2}=\frac{I_{2}}{I_{1}+I_{2}} ; \lambda=2 \pi T_{M} / T_{0} ; \mathrm{t}_{\mathrm{o}}$ is the time of metal gripping by the rolls; $\mathrm{T}_{\mathrm{o}}$ - the period of the system's own oscillations; $\mathrm{t}_{\mathrm{p}}$ - time of the drive's take-off run; $\mathrm{I}_{1}, \mathrm{I}_{2}-$ momentums of electrical motor's rotor and rolls' inertia, respectively; $\omega_{\mathrm{H}}-$ nominal angular velocity of drive's rotation; $\mathrm{T}_{\mathrm{M}}=I_{0} R / c_{e} c_{M}-$ electrical and mechanical constant of drive's time; $\mathrm{I}_{\mathrm{o}}$ - general inertia momentum of the mill's drive; $\mathrm{R}$ - resistance in Ohms within anchor's chain of the electric motor; $c_{e}, c_{M}-$ parameters of the electric motor.

After metal gripping by the rolls, elastic momentum in the drive's line is determined according to the formula [8]:

$$
\begin{aligned}
& M_{12}=M_{n p \mathrm{i}}-n_{2} M_{n p}\left(\lambda^{2} \mathrm{e}^{-\mathrm{t} / \mathrm{T}_{\mathrm{M}}}+\lambda \sin p t_{1}-\lambda^{2} \cos p t_{1}\right) /\left(1+\lambda^{2}\right)+ \\
& +A \cos p t_{1}+B \sin p t_{1}
\end{aligned}
$$

here $M_{n p \mathrm{i}}=n_{1} M_{n p}+n_{2}\left(M_{n p}+\mathrm{I}_{0} \omega_{H} / t_{p}\right) ; t_{1}=t-t_{0} ; n_{1}=I_{1} /\left(I_{1}+I_{2}\right)$;

$$
\begin{aligned}
& A=n_{2} M_{a}\left(1-\cos p t_{0}\right)-M_{n p \mathrm{i}}+M_{n p}\left(1-\sin \mathrm{pt}_{0}\right) / p t_{0}+ \\
& +n_{2} M_{n p}^{\prime}\left(\lambda^{2} e^{-t_{0} / T_{M}}+\lambda \sin p t_{0}-\lambda^{2} \cos p t_{0}\right) /\left(1+\lambda^{2}\right) \\
& B=n_{2} M_{n p} \sin p t_{0}+M_{n p}\left(1-\cos p t_{0}\right) / p t_{0}+ \\
& +n_{2} M_{n p}^{\prime}\left(-\lambda e^{-t_{0} / T_{M}}+\lambda \cos p t_{0}+\lambda^{2} \sin p t_{0}\right) /\left(1+\lambda^{2}\right) .
\end{aligned}
$$

As an example of numerical realization of the designed program means, a rolling process used for a narrow slab $\left(h_{0} \times B_{0}=150 \times 1000 \mathrm{~mm}\right.$, weight 15 tons $)$ was investigated, the parameters of the slab being:

$$
\begin{gathered}
I_{1}=98 t \cdot m^{2} ; I_{2}=5 t \cdot m^{2} ; \mathrm{T}_{\mathrm{M}}=0,06 \mathrm{~s} ; n_{1}=0,95 ; n_{2}=0,05 ; p=122 \mathrm{~s}^{-1} \\
T=0,051 \mathrm{~s} .
\end{gathered}
$$

In Fig. 5 and 6 the specified distribution of the rolling force and momentum were 


\section{TPACEE-2016}

presented, here, the load peaks at the time of gripping and metal exit from the rolls were pointed out.

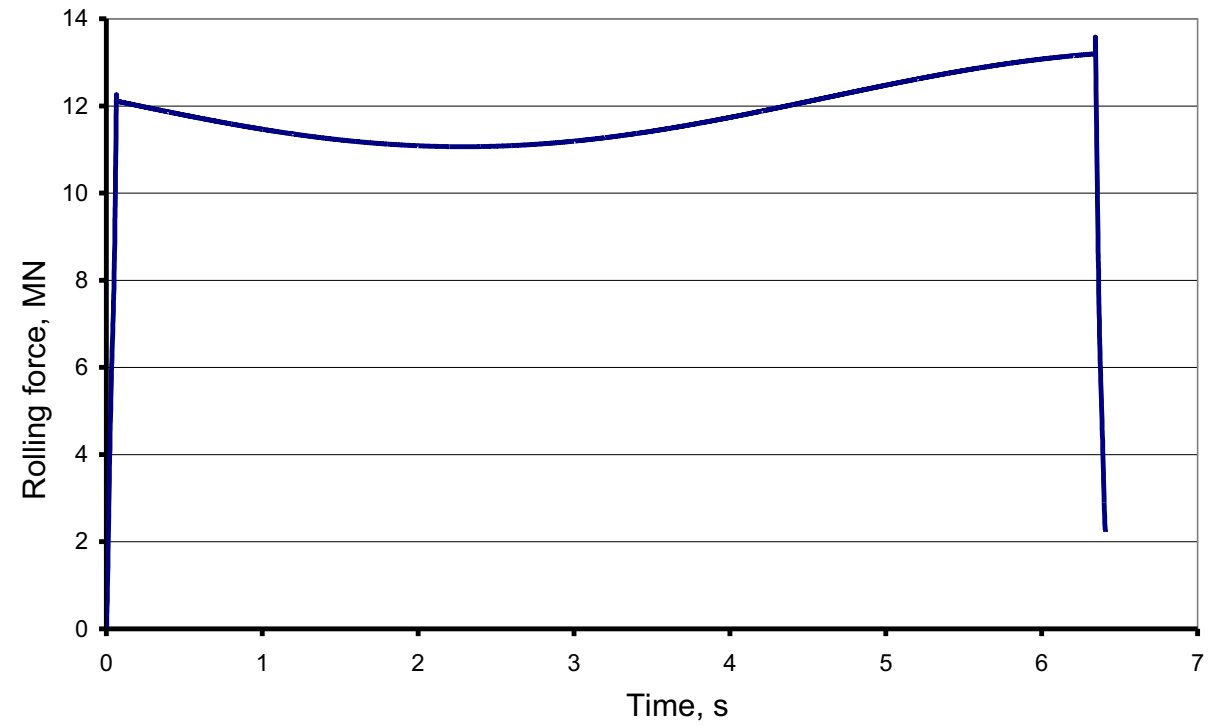

Fig. 5. Distribution of the rolling force.

In Fig. 7 the specified distribution of momentums inside elastic sections of the operating stand were pictured.

It can be seen from the dependences that the coefficient of dynamics at metal gripping was of 1.5 order, and at rolling it was equal to $1.2 \ldots 1.35$ order. The change of the momentum here has a complicated character. Attention should be given to this during the design of the elements of the line of stand's drive.

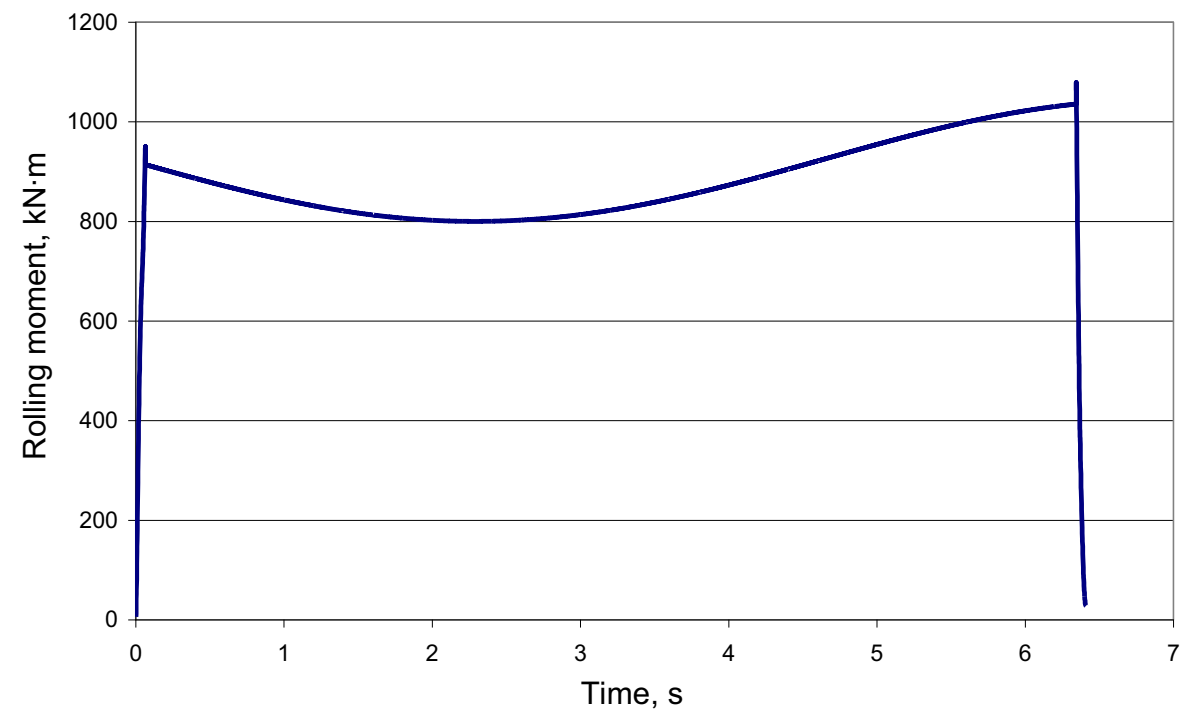

Fig. 6. Distribution of the rolling momentum. 


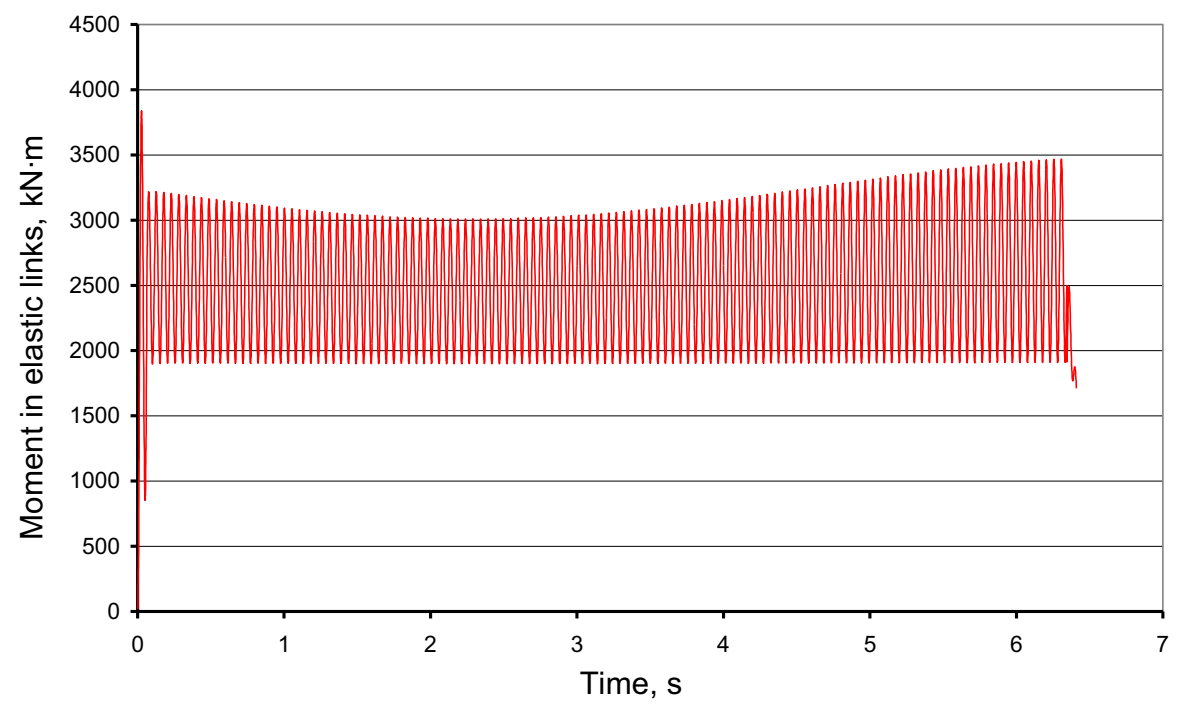

Fig. 7. Distribution of the momentums in elastic sections.

\section{Results and Discussion}

One drawback of the existing dynamic models is the necessity of a simplified dependence of the force and momentum of rolling in time. Correction of the original prerequisites in plasticity condition and analytical description of the coefficient of external contact time allowed us to develop engineering methods of calculating the energy parameters of hot rolling of relatively thin strips, with the relative degree of their error (as compared to more strict two-dimensional methods of analysis) less than 5\%. It is obvious from the analysis of the specified estimate distributions that unlike the methods used in the cited works [20,21], the influence of the external friction coefficient $\mu$ is essential enough even within $\mathrm{L} / \mathrm{h}_{\mathrm{cp}}>1$ range. As an example of the use of the prepared mathematical models for determination of energy parameters of the rolling practice, the results of calculation of changes of dynamic loads in the line of a drive of a blooming mill and also changes in momentum in drive's elastic lines were presented.

\section{Conclusions}

On the basis of two-dimensional FEM approach and numerical recurrent solutions of finitedifferential forms of the condition of static equilibrium of detached elementary volumes, obtained by means of fragmentation of the strip along the rolling line and the analysis of the area of plastic deformation, mathematical models were developed for stressed-deformed metal state at hot rolling of sheets, plates and strips, which made it possible to improve dynamic models of drive's lines of the operating stands, by means of taking into account the real character of loads changes and geometrical parameters, as well as physical and mechanical properties of strip's material at rolling.

The reported study was funded by RFBR according to the research project №16-08-00845a «Verification and development of models of inelastic deformation at the passive loading». The authors acknowledge the assistance given by Ralph Williams who thoroughly edited the final version of the paper in English, making it acceptable for publication. The authors declare that there is no conflict of interest regarding the publication of this paper. 


\section{References}

1. R.S. Adamia, Optimization of dynamic loads of rolling mills (Metallurgy, Moscow, 1978)

2. V. Mazur, V. Artyukh, G. Artyukh, M. Takadzhi, Engineering Designer. 37(1), 26-29 (2012)

3. V.V. Verenev, V.I. Bolshakov, A.M. Yunakov, Fundamental and applied problems of ferrous metallurgy: Works, Dnepropetrovsk. 19, 346-358 (2009)

4. R.A. Yakovlev, Dynamic calculation of rolling mills (Bauman MSTU, Moscow, 1964)

5. V.V. Verenev, V.I. Bolshakov, N.I. Podobedov, Protection of iron and steel machines from failure. 3, 35-39 (1998)

6. F.K. Ivanchenko, V.M. Gribennik, V.I. Shiryaev, Calculation of machines and mechanisms of rolling shops (Higher scool publishers, Kiev, 1995)

7. V.A. Fedorinov, A.V. Satonin, E.P. Gribkov, Mathematical modelling of stresses, deformations and main indices of quality at rolling of relatively wide sheets and strips (DSMA, Kramatorsk, 2010)

8. A.V. Satonin, Improvement of the processes and equipment for plastic working of metals in metallurgy and machine building: Volume of works. 298-302 (2000)

9. A.M. Laptev, Y.Y. Tkachenko, V.I. Zhabin, Plastic working of metals. 3(28), 129-135 (2011)

10. Nabeel S. Gharaibeh, Mohammed I. Matarneh, V.G. Artyukh, Research Journal of Applied Sciences, Engineering and Technology. 8(12), 1461-1464 (2014)

11. V.F. Potapkin, Method of fields of sliding lines in the theory of rolling of wide strips (DSMA, Kramatorsk, 2005)

12. SIMULIA Abacus (CAE User's manual), V.6.7 (2008)

13. L.V. Andreyuk, G.G. Tylenev, Steel. 9, 545-547 (1972)

14. M.Y. Browman, Application of theory of plasticity in rolling practice (Metallurgy publishers, Moscow, 1991)

15. A.I. Tselikov, G.S. Nikitin, S.E. Rokotyan, Theory of longitudinal rolling (Metallurgy publishers, Moscow, 1980)

16. Mohammed I. Matarneh, Nabeel S. Gharaibeh, V. G. Artyukh, International Journal of Engineering Science and Innovative Technology. V.4, Issue 2, 1-7 (2015)

17. V.F. Potapkin, Method of the fields of sliding lines fields in the theory of rolling of wide strips (DSMA, Kramatorsk, 2005)

18. E.A. Nekliudova, A.S. Semenov, S.G. Semenov, B.E. Melnikov, Applied Mechanics and Materials. 725-726, 648-653 (2015)

19. I. Maniak, B. Melnikov, A. Semenov, S. Saikin, Applied Mechanics and Materials. 725-726, 943-948 (2015)

20. Firas M.F. Al-Quran, M.E. Matarneh, V.G. Artukh, Research Journal of Applied Sciences, Engineering and Technology. 4(11), 1585-1589 (2012)

21. V.G. Artiukh, S.Yu. Karlushin, E.N. Sorochan, Procedia Engineering. 117, 938-944 (2015) 